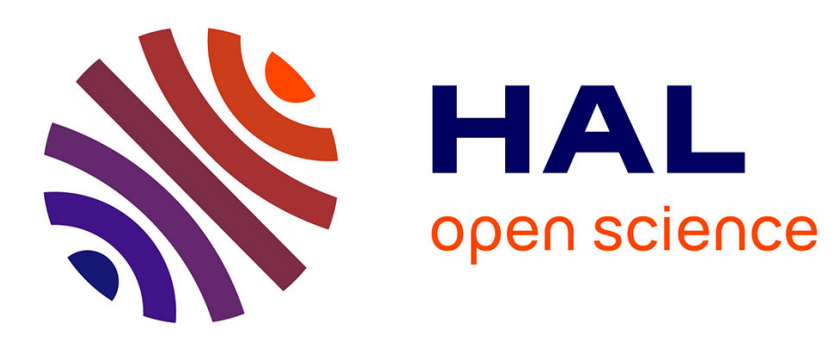

\title{
Geometrically Invariant Watermarking Using Feature Points
}

\author{
Patrick Bas, Jean-Marc Chassery, Benoit Macq
}

\section{To cite this version:}

Patrick Bas, Jean-Marc Chassery, Benoit Macq. Geometrically Invariant Watermarking Using Feature Points. IEEE Transactions on Image Processing, 2002, 11 (9), pp.1014-1028. hal-00166590

\section{HAL Id: hal-00166590 \\ https://hal.science/hal-00166590}

Submitted on 7 Aug 2007

HAL is a multi-disciplinary open access archive for the deposit and dissemination of scientific research documents, whether they are published or not. The documents may come from teaching and research institutions in France or abroad, or from public or private research centers.
L'archive ouverte pluridisciplinaire HAL, est destinée au dépôt et à la diffusion de documents scientifiques de niveau recherche, publiés ou non, émanant des établissements d'enseignement et de recherche français ou étrangers, des laboratoires publics ou privés. 


\title{
Geometrically Invariant Watermarking Using Feature Points
}

\author{
P. Bas, J-M. Chassery * and B. Macq \\ Patrick.Bas@inpg.fr, Jean-Marc.Chassery@inpg.fr, Benoit.Macq@ ele.ucl.ac.be \\ P. Bas, J-M. Chassery: Laboratoire des Images et des Signaux, BP. 46, Saint Martin d'Heres, 38402, \\ France. Tel: +3347682 62 63, Fax: +33476826384 \\ B. Macq: Laboratoire Tele, Batiment Stevin, Place du Levant, B-1348 Louvain-la-Neuve, Belgium. Tel: \\ +32104722 71, Fax: 3210472089 \\ 5-AUTH Authentication and Watermarking
}

\begin{abstract}
This paper presents a new approach for watermarking of digital images providing robustness to geometrical distortions. The weaknesses of classical watermarking methods to geometrical distortions are firstly outlined. Geometrical distortions can be decomposed into two classes: global transformations such as rotations and translations and local transformations such as the StirMark attack. An overview of existing self-synchronising schemes is then presented. Theses schemes can use periodical properties of the mark, invariant properties of transforms, template insertion, or information provided by the original image to counter geometrical distortions.

Thereafter a new class of watermarking schemes using the image content is presented. We propose an embedding and detection scheme where the mark is bound with a content descriptor defined by salient points. Three different types of feature points are studied and their robustness to geometrical transformations is evaluated to develop an enhanced detector. The embedding of the signature is done by extracting feature points of the image and performing a Delaunay tessellation on the set of points. The mark is embedded using a classical additive scheme inside each triangle of the tessellation. The detection is done using correlation properties on the different triangles. The performance of the presented scheme is evaluated after JPEG compression, geometrical attack and transformations. Results show that the fact that the scheme is robust to theses different manipulations. Finally, in our concluding remarks, we analyse the different perspectives of such contentbased watermarking scheme ${ }^{1}$.
\end{abstract}

\section{INTRODUCTION}

During the last decade, digital technologies have grown dramatically. Digital audio, video and software are widely used within home computers and open networks. Nevertheless one particular drawback of digital content is their ability to be volatile and easily processed.

Digital objects may be copied, manipulated or converted easily without any control. The goal of watermarking is to embed an unnoticeable information (called a mark) in the content of media. Thus the functionality of the medium is improved without altering the format.

\footnotetext{
${ }^{1}$ This work was supported by the European IST Aspis project and the National RNRT Aquamars project.
}

Several applications of watermarking have been considered by researchers.

- Copyright: In this case, the mark is associated with an Identity Number. In this way, the mark identifies the owner of the content and can be used for copyright purposes (prevent illegal copies). The embedding and the detection of the mark depend on a secret key. The mark has to be robust and detectable after numerous processing treatments or attacks that preserve the original content.

- Database retrieval and Data hiding: In this case the mark permits the identification of the content. The mark contains a description of the content or a pointer to this description and can be used for database "classification". In this application, the robustness of the mark is not decisive but the mark has to be detected after basic format conversion.

- Authentication: the mark can be a fragile mark that will disappear after image manipulation. It can be used to authenticate the content and to prove that its integrity has been preserved. The function of the mark is to prove that the content has not been manipulated and consequently the robustness of the marking algorithm is not a prior achievement.

\section{A. Problem of synchronization}

The detection of the mark requires a synchronization step to locate the embedded mark in the content. Here are two scenarios that illustrate this idea:

- In audio, the mark is commonly detected in real time. In most practical cases, the position of the beginning of the sequence is not known and cannot be used as a reference. Consequently, the detector must pre-process the audio to know where the mark is located.

- The case of digital images is similar because they can undergo geometrical transformations after analog-to-digital and digital-to-analog conversion such as printing and scanning the image. Resizing and rotating are also basic manipulations in image edition and require a synchronization step for the detection of the mark. 


\section{B. Paper presentation}

In this paper, our work is focused on the problem of mark synchronization in digital images. The basic idea is that, binding the signature with the content of the image, the mark could be detected when the image undergoes geometrical transformations.

We select feature point detectors to represent an image content descriptor. The set of feature point permits image partitioning using a delaunay tessellation. The mark is embedded in each triangle via an affine transformation which allows the orientation of the random sequence. The detection of the mark is based on the same content analysis and is performed using a correlation criterion. The diagram of the presented watermarking scheme is depicted in Figure 1.

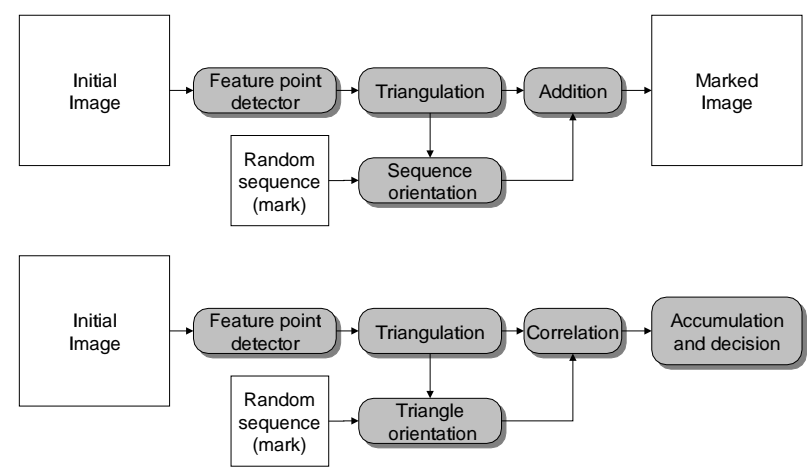

Fig. 1. Outline of the presented content-based watermarking sheme.

The paper is organized as follows: the second section outlines the weaknesses of watermarking schemes against geometrical transformations and geometrical attacks. We also provide an overview of the different watermarking approaches to counter geometrical attacks. We divide the different schemes in several categories to highlight their advantages and limitations. In section 3, we describe a new class of watermarking schemes, called content-based methods, which use image descriptors to orientate the signature. We present a new method, using feature points, that embeds a pre-defined triangular pattern inside triangles in a tessellation of the image. Section 4 presents three feature points detectors and an evaluation of their robustness is presented. In section 5, we describe a basic spatial embedding process. We also present different tests with a large variety of images and different categories of geometrical transformations and compare our results with the Digimarc solution.

Finally, in section 6 we outline the positive aspects and the perspectives offered by the classes of content-based approaches.

\section{Overview of ClassicAl SELF-SYNCHRONISING TECHNIQUES}

The goal of this section is to describe the problem of geometrical distortions and to give an overview of techniques countering geometrical transformations: self-synchronising schemes. We will focus this short overview on four classes of selfsynchronising techniques:

- techniques using periodical sequences,

- techniques using templates insertions,
- techniques using invariant transforms,

- techniques using the original image.

\section{A. Effect of geometrical distortions}

One major drawback of classical watermarking schemes is the lack of robustness to geometrical distortion. In classical additive (spread-spectrum) methods such as presented in [40][36][9] and substitutive methods such as presented in [4][42][25][30], the image is processed as a classical array of pixels with no reference except the coordinates of each pixel. Consequently if the image undergoes a geometrical transformation, the mark in the image cannot be detected without identifying the transformation.

Using an additive scheme, the correlation cannot be performed because the generated random sequence and the embedded random sequence are not synchronized.

Using a substitutive scheme, the location of the marked components cannot be performed because initial locations depend on external coordinates; consequently the decoding of the mark is not possible.

Basically, two levels of geometrical distortions can be outlined:

- The typical geometrical transformation commonly used in image edition such as rotation, translation, cropping, and composition. These transformations are applied on the whole image, and in many ways can be easily represented by a mathematical operation. One basic solution to identify the transformation is to perform an exhaustive detection considering all possible geometrical transformations of the marked image. In this case the computing cost will drastically increase. For example, if we consider only a composition of rotation and scaling operations from $50 \%$ up to $200 \%$ of the image size, the processing cost is roughly multiplied by $5.4 \times 10^{4}$.

- The other category of geometrical distortions are especially designed to desynchronise the mark without visual changes. M. Khun and F. Petitcolas [35] developed a benchmark called StirMark containing different attacks. One of the first developed attacks of this program is composed of local random geometrical distortions that permits us to defeat many classical watermarking schemes without visible alterations [15] (cf Fig. 2).

\section{B. Periodic insertion of the mark}

One strategy to counter the problem of geometrical desynchronization is to add redundancy during the embedding process. This redundancy can be used to localize the position of the signature and to improve the detection phase. We present different methods based on a periodic insertion of the mark.

Kalker and Janseen developed a spatial spread-spectrum scheme for video wartermarking [22].

The authors design a periodic insertion of the mark to cope with the important problem of image shifting in video sequences. A random basis sequence $W_{b}$ of size $L_{b} \times H_{b}$ is repeated to provide a tilling which is added to the original image. The 


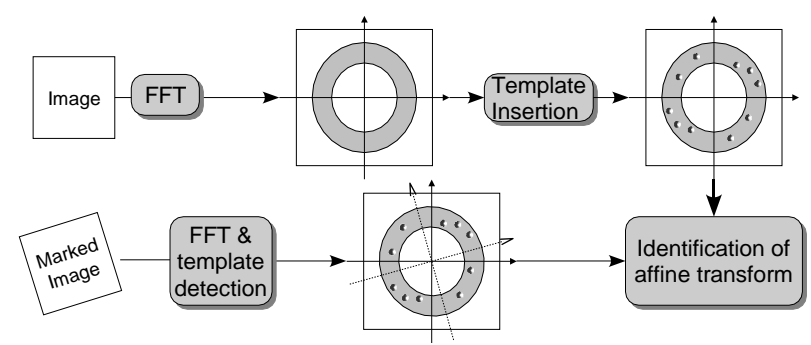

Fig. 6. Principle of template insertion: each template is embedded in the Fourier domain in middle frequencies of the image (upper part of the figure). The identification of the affine transform is performed after a search of template location (lower part of the figure).

faculty to address synchronization of local and global transformations, but attacks have been developed to erase template presence [41].

\section{Invariant transform}

Another way to cope with resynchronisation problems after affine transforms is to embed the watermark in an invariant domain.

Ó Ruanaidh and Pun introduced a watermarking scheme exploiting some properties of the Fourier transform. In this scheme, mark embedding is performed using a RST (Rotation, Scaling and Translation) invariant domain [33].

Given a $2 \mathrm{D}$ function $f\left(x_{1}, x_{2}\right)$ representing the image, the authors extended the Fourier transform invariance properties to cyclic translations using a log-polar mapping:

$$
x=e^{\mu} \cos \theta \quad y=e^{\mu} \sin \theta
$$

where $\mu \in \Re$ and $\theta \in[0 ; 2 \pi[$. Applying the mapping, the rotation of the point $(x, y)$ by an angle $\delta$ and the scaling operation with a factor $\rho$ are considered as a translation:

$$
\begin{gathered}
(\rho x, \rho y) \leftrightarrow(\mu \log \rho, \theta) \\
(x \cos \delta-y \sin \delta, x \sin \delta+y \cos \delta) \leftrightarrow(\mu, \theta+\delta)
\end{gathered}
$$

Applying the Fourier transform after the log-polar mapping provides a translation, rotation, and scaling-invariant domain. This transform is named the Fourier-Mellin Transform. The different stages used to obtain the embedding domain are shown in figure 7.

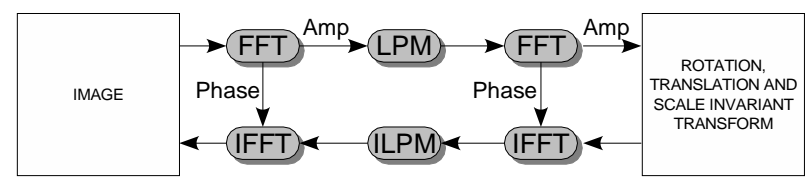

Fig. 7. Composition of transforms used to obtain a rotations, translations and scaling invariant embedding domain. FFT and IFFT denote respectively the Fast Fourier Transform and its inverse. LPM and ILPM denote respectively the log-polar mapping and its inverse.

Lin et al. developed another RST invariant scheme depending on the log-polar coordinates of the Fourier transform [28].
A 1D signal is generated summing the log-magnitude of the 2D log-polar spectrum over a band of radii:

$$
g(\theta)=\sum_{j} \log \left(\left|I\left(\rho_{j}, \theta\right)\right|\right) \quad 0^{\circ} \leq \theta<180^{\circ}
$$

where $I\left(\rho_{j}, \theta\right)$ represents the $2 \mathrm{D}$ log-polar spectrum. The authors outline that the created component $g(\theta)$ is invariant to both translation and scaling operations while rotations generate a circular shift of the signal.

The signature, generated with a mixing function, is added to $g(\theta)$ to create the marked image.

\section{E. Using the original image}

Non-blind watermarking schemes use the original image to detect the mark. These algorithms may use this valuable information to identify the geometrical transformation applied on the image.

Davoine et al used the original image to compensate geometrical deformations induced by the StirMark attack [11].

A regular triangular tessellation is applied on the original image and on the marked and attacked image. The compensation of geometrical distortion is performed by slightly shifting different vertices of the attacked image tessellation (cf Fig. 8). The geometrical compensation can also be done using more basic image feature such as image edges. Once the geometrical compensation has been processed, the mark can be recovered and extracted.
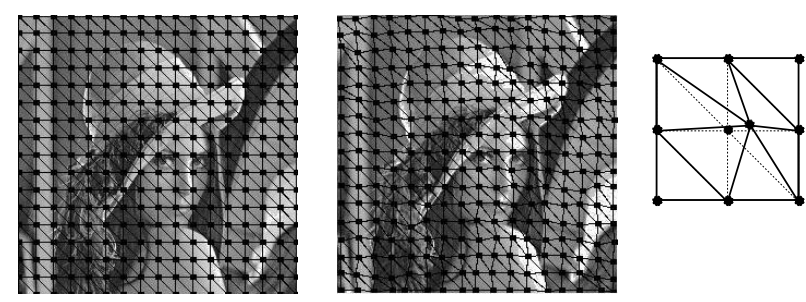

Fig. 8. Left image: original image with triangular tessellation. Right image: image attacked by StirMark after geometrical compensation. Each vertex of the tessellation is slightly moved to minimize quadratic error between each original triangle and the corresponding attacked triangle (right figure).

\section{F. Overview remarks}

We have presented different self-synchronizing schemes that allow the detection of the mark after a special category of geometrical distortions such as translations, general affine transforms or local distortions such as the StirMark attack. It is important to point out that the presented methods are not robust to each category of geometrical distortion.

On one hand, periodic insertion, invariant transforms and template embedding enable synchronization after affine transforms. On the other hand, the use of the original image improves the robustness to local distortions, but the extension to affine transforms implies an increase of the computational cost. Template insertion can address both local and global transformations, but 


\begin{tabular}{|c|c|c|c|}
\hline & $\begin{array}{c}\text { Local transformation } \\
\text { robustness }\end{array}$ & $\begin{array}{c}\text { Global transformation } \\
\text { robustness }\end{array}$ & notes \\
\hline Periodic insertion & no & yes & - \\
\hline Template insertion & no & yes & can be removed \\
\hline Invariant transform & no & yes & - \\
\hline Non-blind & yes & yes & computational cost \\
\hline
\end{tabular}

COMPARISON OF THE PRESENTED METHODS.

this artificial embedding can be easily removed [41].

The different limitations of the presented methods are summed up on Table I.

\section{Content Based Watermarking Schemes}

The objective of this section is to present a new class of watermarking schemes that achieve watermark recovery after geometrical transformations using image content. In particular, the design of a new method using feature points as a content descriptor is presented.

\section{A. Previous works}

Content-based watermarking schemes provide another solution to counter geometrical distortions. In this way the location of the mark is not linked with image coordinates, but with image semantics. The problem of geometrical synchronization is solved because the image content represents an invariant reference to geometrical transformations (cf Fig.9). Content based techniques belongs to second-generation watermaking schemes defined by Kutter et al because the image's content is exploited for the embedding of the mark[27].

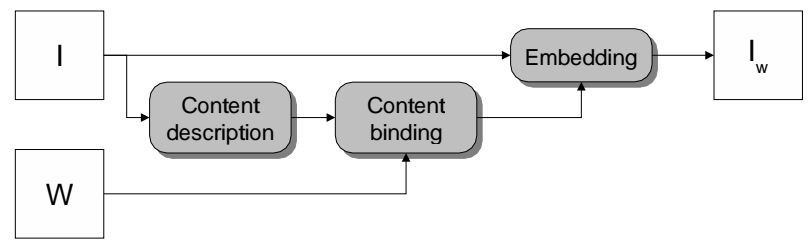

Fig. 9. Principle of a content based watermarking schemes.

Let us start by an overview of different content-based methods.

- Duric et al have proposed a method for recognizing geometrically distorted images and restoring their original appearances [16]. The restored image is used to recover watermarks that were embedded. Image's content is used, represented by feature points, to identify the geometrical transformation. The location of feature points between the original and marked/distorted image permit the identification of the transformation. Local geometric distortions are countered calculating normal displacement fields between the two images. Translation, scaling and rotations are estimated by an affine transform. The presented scheme is robust to the Stirmark attack but the recovery of the mark requires the original image or the initial locations of the feature points.

- Sun et al developed another watermarking method based on image feature to identify the geometrical transformation [39].

Firstly, feature points are extracted from the original and the marked image. Secondly, the synchronizing scheme performs a matching between the two set of points and identify the transformation (cf Fig. 10).

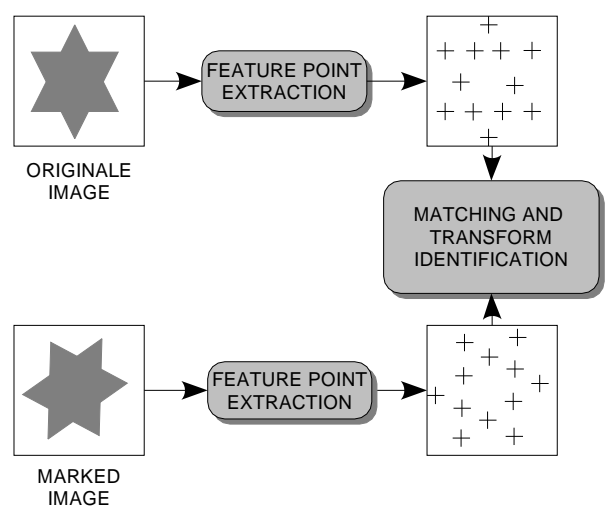

Fig. 10. Principle of geometric synchronization based on feature points extraction.

- Alghoniemy and Tewfik introduced a RST synchronization scheme based on the wavelet decomposition of the image [1].

Wavelet maxima are extracted from low frequency components of the wavelet image decomposition. The scale factor $\gamma$ is estimated by computing the average distance of each maximum (i.e. $\sigma$ ) from the gravity center of the extracted maximum i.e. $\left(x_{0}, y_{0}\right)$ :

$$
\sigma=\sqrt{\frac{1}{N} \sum_{i=1}^{N}\left(x_{i}-x_{0}\right)^{2}+\left(y_{i}-y_{0}\right)^{2}}
$$

Hence, the scale factor $\gamma$ is given by:

$$
\gamma=\frac{\sigma_{s}}{\sigma_{o}}
$$

where $\sigma_{s}$ and $\sigma_{o}$ represent respectively the average distances considered on the marked image and on the original image. The rotation angle estimate $\theta$ is given calculating the difference between the sums of angles in the first quadrant of the wavelet decomposition for the 
original $\left(\theta^{o}\right)$ and the marked image $\left(\theta^{r}\right)$ :

$$
\theta=\frac{1}{N}\left(\sum_{i=1}^{N} \theta_{i}^{o}-\sum_{i=1}^{N} \theta_{i}^{r}\right)
$$

- Dittman et al have designed a content-based watermarking method that does not require the original image and uses self spanning pattern [13]. A basic pattern is represented by a polygon that is spanned over four image inherent points like image edges or corners. The other patterns are spanned around the basic pattern and each pattern carries one bit of the whole watermark (cf Fig. 11). Finally the mark detection is done after recovering feature points locations. Experimental results, presented for a large range of geometrical transformations, illustrate the fact that the presented algorithm is quite robust to geometrical distortions.

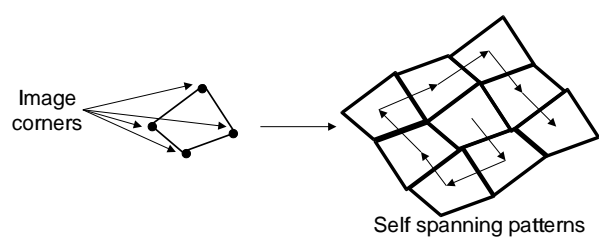

Fig. 11. Self spanning pattern generation.

\section{B. Method design}

To develop a content-based method, we first have to select an appropriate tool to extract an image content descriptor. This representation has to be robust to geometric transformations. We orient our choice towards tools commonly used in pattern recognition: feature points detectors. Our first approach was initially proposed in [3].

Our proposed system can be classically dissociated into two different steps, the embedder and the detector. An overview of the different stages is summarized below:

In the embedder side:

1) Detect robust feature points in an image.

2) Generate a triangular tessellation of the image based on the set of feature points.

3) Map a triangular spread sequence (watermark) into each triangle of the tessellation via affine transform.

4) Add the mapped sequence on each triangle.

In the detector side:

1) Reconstruct the tessellation.

2) Map each triangle to the shape of the original triangular watermark.

3) Compute the correlation of each mapped triangle with the original watermark.

4) Accumulate the correlations to detect the watermark over the whole image.

Consequently the next section (section IV) of this paper is devoted on an evaluation of different feature point detectors, and the content-based watermarking scheme is presented in section V.

\section{FEATURE POINTS DETECTORS}

The objective of this section is to give an overview of different feature points detectors and to present a benchmark system for selecting a robust detector.

It is well known that feature points detectors find salient points in natural images. These points are often located near corners and edges of the image. Feature points were first developed for computer vision and reconstruction [6][17] but they are also employed in data-base retrieval as a descriptor of the image [29][14].

We focus our study on three detectors that are commonly used in pattern recognition and vision systems.

\section{A. Harris Detector}

The Harris and Stephens detector was developed for 3-D reconstruction [19]. This detector uses differential features of the image.

The construction of the detector is based on a corner detection function created by Moravec [32]. The response function $E_{x, y}$ is calculated for a shift $(x, y)$ from the central point $(u, v)$ :

$$
E_{x, y}=\sum_{u, v} w_{u, v}\left|I_{x+u, y+v}-I_{u, v}\right|^{2}
$$

where $I_{u, v}$ represents the luminance of the image at the coordinate $(u, v)$. the function $w_{u, v}$ represents a rectangular window centred on $(x, y)$.

Harris reformulated the detection function using a matricial formulation.

Given:

$$
\begin{aligned}
X & =I *[-1,0,1] \approx \delta I / \delta x \\
Y & =I *[-1,0,1]^{T} \approx \delta I / \delta y \\
A & =X^{2} * w \\
B & =Y^{2} * w \\
C & =(X Y) * w
\end{aligned}
$$

where $*$ denotes the convolution product. The detection function $E_{x, y}$ is then expressed by:

$$
E_{x, y}=(x, y) M(x, y)^{T} \quad \text { with } \quad M=\left[\begin{array}{ll}
A_{x, y} & C_{x, y} \\
C_{x, y} & B_{x, y}
\end{array}\right]
$$

$E_{x, y}$ can be considered as a local auto-correlation function of the image with a shape factor $M$. Harris and Stephens gave a new definition of the detector function of the eigenvalue $\alpha$ and $\beta$ of the array $M$. These values are invariant by rotation and if their magnitudes are high, the local auto-correlation function is represented by a local peak.

To avoid computing the eigenvalue of $M$, the new criterion is based on the trace and determinant of $M$ :

$$
\begin{aligned}
\operatorname{Tr}(M) & =\alpha+\beta=A+B \\
\operatorname{Det}(M) & =\alpha \beta=A B-C^{2} \\
R_{H} & =\operatorname{Det}(M)-k \operatorname{Tr}^{2}(M)
\end{aligned}
$$


where $k$ is an arbitrary constant.

Feature points extraction is achieved by applying a threshold on the response $R_{H}$ and searching for local maxima.

$$
\begin{gathered}
\{(i, j)\} \backslash R_{H}(i, j)>\eta \quad \text { and } \\
\forall(u, v) \in V_{i, j}, R_{H}(i, j) \geq R_{H}(u, v)
\end{gathered}
$$

The function $R_{H}$ applied on lena is shown on Figure 12.

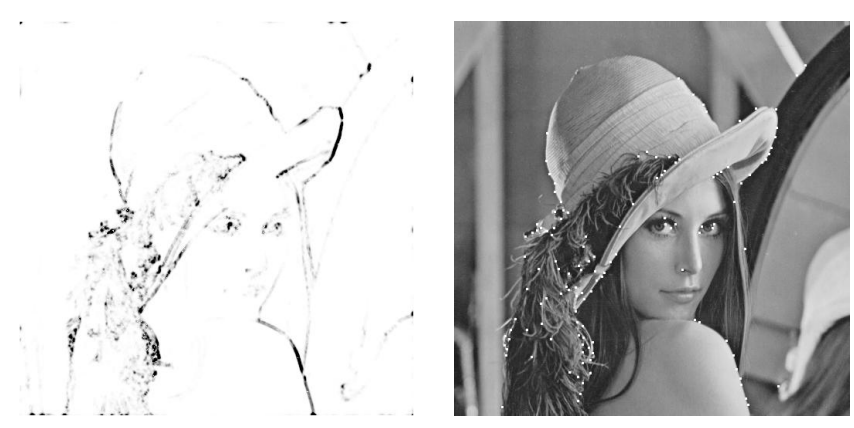

Fig. 12. Harris detector response.

\section{B. Other tested detectors}

We have also tested two other detectors: the Achard-Rouquet [7] detector and the SUSAN detector [38].

The Achard-Rouquet detector is based on the fact that the angle between two gradient vectors is important around corners.

The corner strength is hence calculated using cross product of the neighbour gradients.

$$
\begin{aligned}
& k_{i, j}= \sum_{u, v \in V_{i, j}}\left\|\overrightarrow{\operatorname{grad}}\left(I_{i, j}\right) \wedge \overrightarrow{\operatorname{grad}}\left(I_{u, v}\right)\right\|^{2} \\
&= \sum_{u, v \in V_{i, j}}\left\|\overrightarrow{\operatorname{grad}}\left(I_{i, j}\right)\right\|^{2} \cdot\left\|\overrightarrow{\operatorname{grad}}\left(I_{u, v}\right)\right\|^{2} \\
& \sin ^{2}\left[\overrightarrow{\operatorname{grad}}\left(I_{i, j}\right), \overrightarrow{\operatorname{grad}}\left(I_{u, v}\right)\right]
\end{aligned}
$$

where $V_{i, j}$ represents the neighbourhood of the pixel $(i, j)$ and $\cdot$ denotes the cross product. Given :

$$
\begin{aligned}
& X=\delta I / \delta x \\
& Y=\delta I / \delta y
\end{aligned}
$$

and developing the equation 17 , the authors obtained a normalized version of the detector

$$
k=\frac{I_{x}^{2}<I_{y}^{2}>+I_{y}^{2}<I_{x}^{2}>-2 I_{x} I_{y}<I_{x} I_{y}>}{<I_{x}^{2}>+<I_{y}^{2}>}
$$

where $<>$ denotes the mean applied on the neighbourhood $V_{i, j}$.

The set of feature points is obtained using a threshold of the value $k$ and selecting local maximal.

The SUSAN detector is based on a geometrical observation of the image [38]. SUSAN means Smallest Univalue Segment Assimilating Nucleus.
In this method, each pixel of the image, representing a nucleus, is associated with a circular mask. The mask is divided in two distinct areas, the USAN area (Univalue Segment Assimilating Nucleus) and its complement.

A corner of the image is consequently represented by a local minimum of the USAN function applied on the image. The principle of this detector is illustrated on figure 13 .

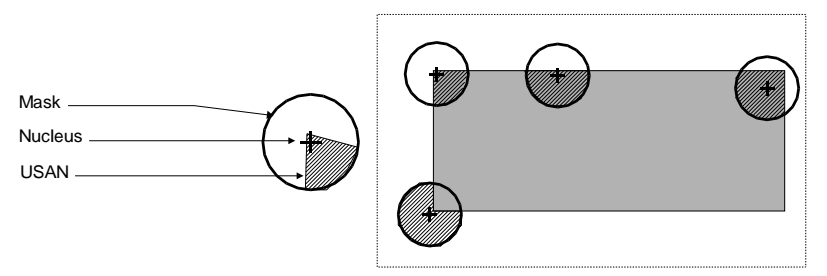

Fig. 13. SUSAN detector principle.

The number of pixels that belong the USAN area allows the computation of the detector magnitude. The USAN computing is done by calculating $n\left(I_{i, j}\right)$ :

$$
n\left(I_{i, j}\right)=\sum_{u, v \in V_{i, j}} C(i, j, u, v)
$$

The $C(i, j, u, v)$ function represents the mask geometry. A feature point is consequently detected if $n\left(I_{i, j}\right)$ is smaller than nbMax. nbMax representing half of the total number of pixels in the mask.

\section{Detector benchmark}

The next step of this study is logically to evaluate of the robustness of these different detectors. To this end, we designed a function named score that evaluates the capacity of the detector to preserve the selected points when the image undergoes a geometrical transformation or other processing.

This function is defined as follows:

$$
\begin{gathered}
\text { score }=\frac{N b_{p r e}-\left(N b_{c r e}+N b_{d e s}\right)}{N b_{p r e}+N b_{d e s}} \\
=\frac{N b_{p r e}-\left(N b_{c r e}+N b_{d e s}\right)}{N b_{i n i}}
\end{gathered}
$$

where $N b_{i n i}$ denotes the number of points present in the initial image, and $N b_{\text {cre }}, N b_{d e s}, N b_{\text {pre }}$ represent respectively the number of points that have been created, destroyed and preserved after the process. This function is maximal when $N b_{\text {cre }}=N b_{\text {des }}=0$, and is in this case equal to 1 .

We evaluated this function on four different images:

- The $512 \times 512$ lena image, is well known in image processing. This is a high-contrast image with sharp corners and textured areas.

- The $512 \times 512$ baboon image. This image contains a large textured area (the fur of the animal) and a homogeneous area represented by the face.

- The $454 \times 652$ tree image is an extremely textured image in which the geometrical singularities are visually hard to designate.

- The $549 \times 748$ water image belongs to the JPEG2000 database. This is an image with large homogeneous areas 
and its content is indistinct and poor.

The score was evaluated after registering feature points and computing the inverse transformation to compute the counts. We decided also to evaluate the function score on 25 different processes.

- 10 rotations with different angles $\left(20^{\circ}, 40^{\circ}, 60^{\circ}, 80^{\circ}\right.$, $100^{\circ}, 120^{\circ}, 140^{\circ}, 160^{\circ}, 180^{\circ}, 200^{\circ}$ et $220^{\circ}$ ). We applied after each rotation an inverse rotation to compute the score.

- 5 scaling operations, with a 0.9 down to 0.5 factor. We performed the inverse transformation to compute the score.

- 5 Jpeg compressions with a $90 \%$ down to $50 \%$ quality factor.

- 5 noise additions. We added an uniform noise with peak values equal from \pm 50 to \pm 10 for 256 grey level images.

The final score is given by calculating the mean of the different scores after each operation.

\section{Enhanced Detector}

We decided to use to a pre-filtering blur operation because a feature point detector is sensitive to image variation such as corners or textured areas. If the content of the image is textured or noisy, the potential number of feature points becomes important and the robustness of the detector is reduced.

We used a $n \times n$ mask represented by:

$$
M_{n}=\left[\begin{array}{ccc}
1 & \cdots & 1 \\
\vdots & \ddots & 1 \\
1 & \cdots & 1
\end{array}\right]
$$

The figure 14 represents the computed score for different sizes of $n$. We can see that this pre-filtering operation increases the robustness of the detectors. In the rest of this study, we decided to choose a $5 \times 5$ filter which offers the best trade-off between the robustness and the processing time.

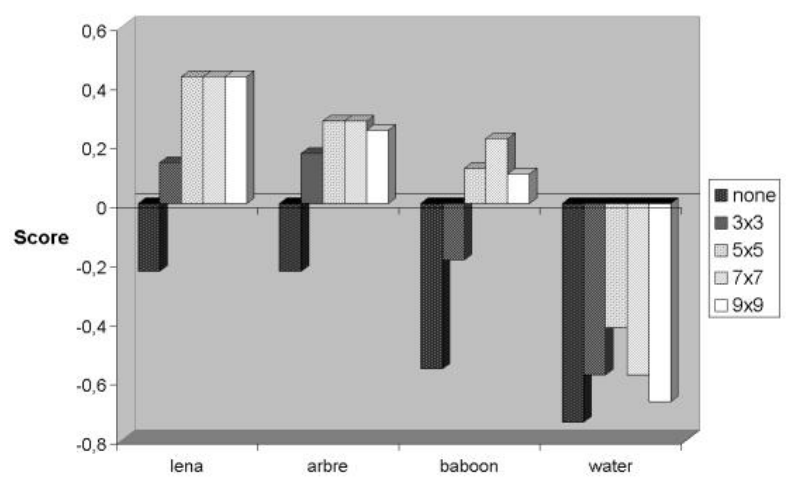

Fig. 14. Blurring filtering and the robustness of the Harris detector.

A feature point is represented by a local maximum of the detector response. It is also important to define the size of the neighbourhood. If this size is too small, the distribution of the different feature points is concentrated on textured areas. If the size of neighbourhood is too large, the feature points become isolated.

To obtain an homogeneous distribution of feature points in the image, we chose to use a circular neighbourhood to avoid increasing detector anisotropy. The centre of the neighbourhood is the considered pixel. To be robust to scaling operations, the circle diameter depends on the image dimensions:

$$
D=\frac{w+h}{\gamma}
$$

The integers $w$ and $h$ represent respectively the image width and height. The neighbourhood size is quantized by the $\gamma$ value.

Figure 15 illustrates the influence of the $\gamma$ parameter. We decided to choose a $\gamma$ value equal to 24 to obtain an homogeneous repartition of the feature points.

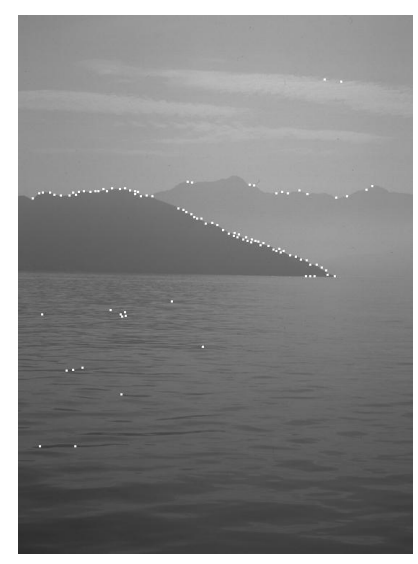

$\gamma=200$

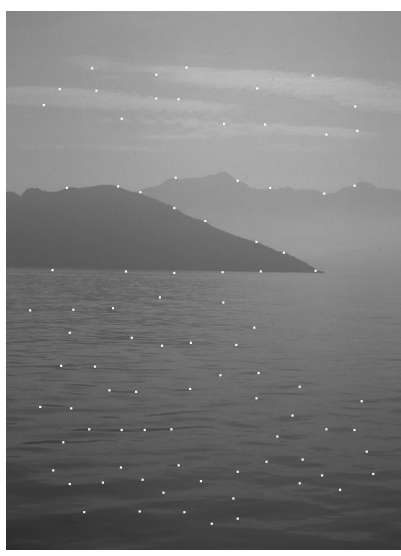

$\gamma=24$
Fig. 15. $\gamma$ parameter effects.

\section{E. Selecting the best detector}

We calculated the detectors' scores on the four test images. These different scores are presented on figure 16. From these results we can deduce two important elements:

- The Harris detector is the most robust detector on each image.

- The detector robustness depends on the content of the image. The score is the highest for the lena image which contains sharp corners. For textured images as baboon or tree, the score is less important. The weakest result is obtained for the water image. This is mainly due to the fact that the content of this image can be greatly modified by noise addition or Jpeg compression.

\section{EMBEDDING AND DETECTION SCHEMES}

The objective of this section is to show how feature points are used in this content-based watermarking method. The embedding and detection scheme are presented and the performance of our method are described. 


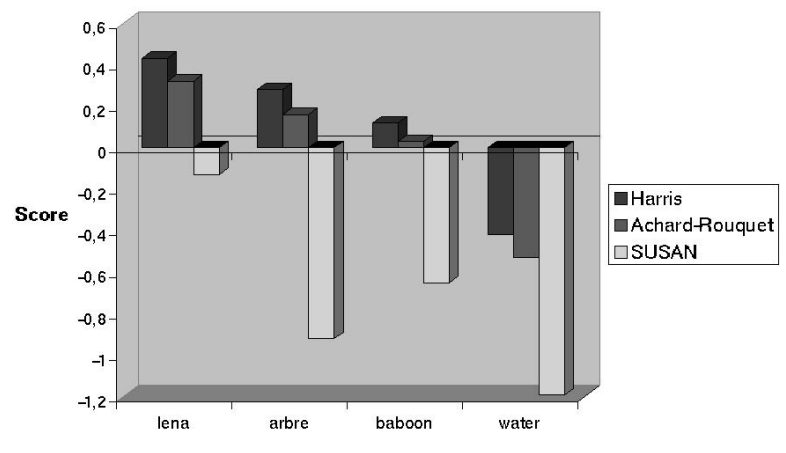

Fig. 16. Behavior of the selected detectors towards our benchmark for the different test images.

\section{A. Embedding scheme}

Let us start by an overview of the embedding part. Firstly, the mark embedding uses references provided by feature point detectors. Next, a Delaunay tessellation is employed to decompose the image into a set of disjoint triangles. The signature embedding is done by inserting a mark inside each triangle of the tessellation.

We focus our choice on Delaunay tessellation because it brings important properties:

- The tessellation has local properties: if a vertex disappears, the tessellation is only modified on connected triangles

- Each vertex is associated with a stability area in which the tessellation is not modified [5] when the vertex is moving inside this area.

- The computational cost is low: a Delaunay tessellation can be done using fast algorithms [8][37].

The general embedding scheme is shown in figure 18. Let us decompose this scheme into different steps:

1) A random sequence $T_{w}$ is generated. The shape of this sequence is delimited by a right-angled isosceles triangle with an arbitrary size (in our tests $64 \times 64$ ). A seed initialising the generator defines the secret key. The sequence values belong to the set $\{-1,+1\}$ and the mean of sequence is zero.

We chose to spread the sequence on $2 \times 2$ pixel blocks. This allows both improving the signature detection after the blurring process, and offering a better resilience to feature point positioning errors.

2) The detection of feature points using our improved Harris detector is applied to the image. We obtain a set of points $P=\left\{p_{i} \in \mathbb{R}^{2}, i=1, \ldots, n\right\}$.

3) A Delaunay tessellation $T=\left\{T_{i}\right\}, 0 \leq i<N$ is performed using $P$.

The Delaunay tessellation of the set $P$ is defined to be the unique triangulation of the convex cover of $P$ such as the interior of the containing circle $\mathcal{C}\left(p_{i}, p_{j}, p_{k}\right)$ of each triangle $\left(p_{i}, p_{j}, p_{k}\right)$ of $P^{3}$ does not contain another vertex of $P$ :

$$
\begin{gathered}
\operatorname{Del}(P)=\left\{\left(p_{i}, p_{j}, p_{k}\right) \in P^{3} \backslash\right. \\
\left.\mathcal{C}\left(p_{i}, p_{j}, p_{k}\right) \cap\left(P-p_{i}-p_{j}-p_{k}\right)=\emptyset\right\}
\end{gathered}
$$

The constructed triangle defined a Delaunay triangle. The signature detection is performed in each triangle $T_{k} \in T$.

4) An affine transform is performed on triangle $T_{w}$ to map the shape of $T_{k}$. The affine transform is oriented using the magnitude of each angle issued from $T_{k}$. We use a splinecubic interpolation during the transformation to preserve high frequencies of $T_{w}$ [31][24]. The triangle $T_{m}$ is then obtained.

The affine function $\mathcal{A}$ allows us to transform a point $\left(x_{w}, y_{w}\right)$ of the triangle $T_{w}$ into a point $\left(x_{m}, y_{m}\right)$ of the triangle $T_{m} . \mathcal{A}$ is defined by six real parameters $a, b, c, d$, $e$ and $f$. The relation between two pairs of points is given by:

$$
\begin{gathered}
\mathcal{A}\left(x_{w}, y_{w}\right)=\left(\begin{array}{l}
x_{m} \\
y_{m}
\end{array}\right)= \\
{\left[\begin{array}{ll}
a & b \\
c & d
\end{array}\right]\left(\begin{array}{l}
x_{w} \\
y_{w}
\end{array}\right)+\left(\begin{array}{l}
e \\
f
\end{array}\right)=L\left(\begin{array}{l}
x_{w} \\
y_{w}
\end{array}\right)+T}
\end{gathered}
$$

This affine transform $\mathcal{A}$ permits us to model geometrical transformations such as rotations, scaling operations, or shearing effects.

There are six different affine transforms that map the triangle $T_{w}$ into the triangle $T_{m}$. The selected affine transform has to be unique. Consequently, we decided to classify the different vertices of $T_{m}$. We use the magnitude of the angles to obtain a unique transformation (cf Fig 17).
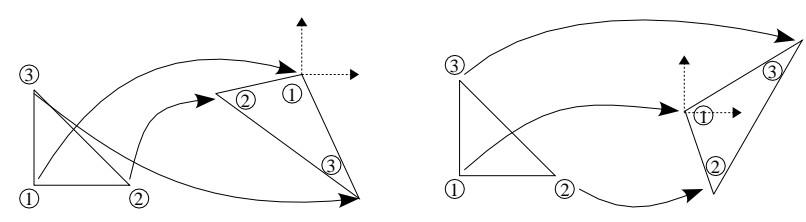

Fig. 17. Orientation of the triangle.

5) $T_{m}$ is multiplied by a visual mask. This mask, named $\Lambda$, is based on the image luminance, We exploit the fact that the human eye is less sensitive to contrast in dark or bright areas than to middle luminance areas [23].The signature $T_{p}$ is obtained by weighting $T_{m}$ :

$$
T_{p}(i, j)=\alpha \times \Lambda(i, j) \times T_{m}(i, j)
$$

The signature power is adjusted using the real $\alpha$.

6) The marked triangle is obtained adding $T_{p}$ and $T_{k}$ : $T_{i}=T_{p}+T_{k}$.

7) $T_{i}$ is substituted for $T_{k}$. 


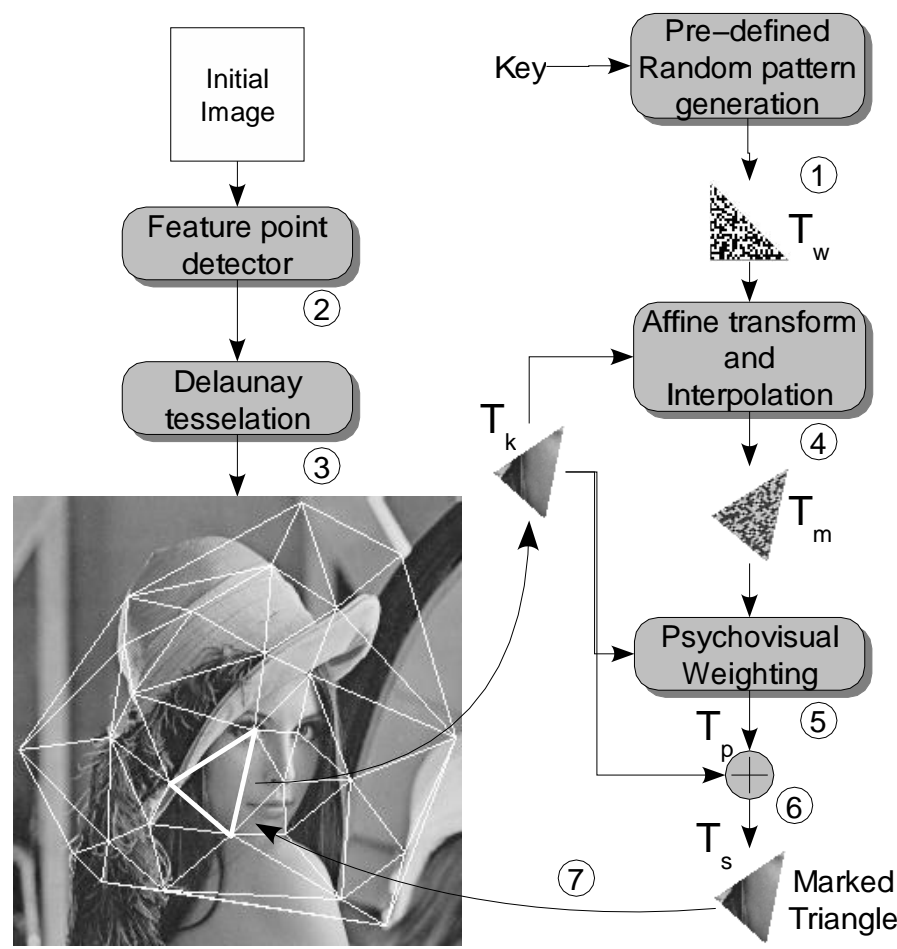

Fig. 18. Embedding process in our proposed watermarking scheme.

\section{B. Detection scheme}

The detection process uses the result of the feature point detection and consequently performs a self-synchronisation of the mark. If the marked image undergoes a geometric transformation, the set of salient points mainly follows the transformation and several triangles are consequently conserved. The presence of the signature in a triangle is certified by a correlation criterion.

The detection scheme is illustrated on figure 21. We now detail the different steps of the signature detection; the three first steps are identical to the insertion scheme:

1) $T_{w}$ is generated from a random sequence depending on a secret key.

2) The detection of feature points using our improved Harris detector is applied to the image.

3) A Delaunay tessellation $T=\left\{T_{i}\right\}, 0 \leq i<N$ is performed using image feature points. The mark is inserted in each triangle $T_{k} \in T$.

4) Each triangle $T_{k}$ is warped into a $64 \times 64$ right-angled isosceles triangle denoted $T_{\mathrm{L}}$.

5) From $T_{\llcorner}$we process a Wiener filtering using $T_{w}$ statistics to eliminate components provided by the original image. We obtain $\hat{T}_{w}$ :
$\hat{T}_{w}(i, j)=\frac{V_{l}\left[T_{w}\right](i, j)}{V_{l}\left[T_{w}\right](i, j)+V_{l}\left[T_{\llcorner}\right](i, j)}\left[T_{\llcorner}(i, j)-M_{l}\left[T_{\llcorner}\right](i, j)\right]$

$V_{l}()$ represents the local standard variance and $M_{l}()$ the local mean for the $(i, j)$ location.

Wiener prediction can be considered as a denoising operation [21] and allows separation of the image components from the mark components. In case of textured areas, the signal-to-noise ratio is lower, and consequently the signature detection, computing the correlation between $T_{w}$ and $T_{\mathrm{L}}$, is perturbed by the image components. Figure 19 represents effects of Wiener filtering.

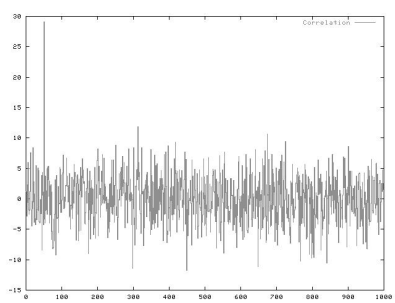

correlation values using Wiener prediction

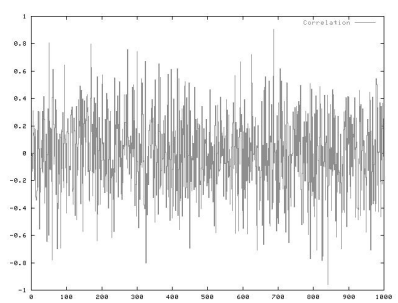

correlation values without

Wiener prediction
Fig. 19. Effects of Wiener prediction: the correlation is computed on a mark triangle with a key equal to 50 . The chosen triangle is textured. On one hand Wiener filtering enables the detection of the signature (left curve). On the other hand, using classical correlation, we cannot detect the presence of the signature.

6) The signature detection can be performed using two different levels. First it can be done locally by comparing the magnitude of $\operatorname{corr}\left(T_{w} ; \hat{T}_{w}\right)$ with a threshold for each triangle. The threshold depends on a false response probability. We represent the statistics in a case of a false response by a normal distribution.

A mark can only be detected according to a probability of false alarm $P_{f a}$ representing the probability of signature detection in a image which does not contain the mark.

In our study, the signature is detected inside a triangle if:

$$
\operatorname{corr}\left(T_{w} ; \hat{T}_{w}\right) \geq \eta\left(P_{f a}\right)
$$

where the real $\eta\left(P_{f a}\right)$ represents a threshold depending on the false-alarm probability. To estimate the value of $\eta\left(P_{f a}\right)$, we performed an estimation of the random variable $X_{k}=\operatorname{corr}\left(T_{w 1}, \hat{T}_{w k}\right)$ assuming that $k \neq 1$.

With the assumption that $X$ is represented by a sum of independent elements (due to the correlation), we decided to express the distribution of $X$ as a gaussian law $\mathcal{N}(m, \sigma), m$ and $\sigma$ representing respectively the mean and variance estimation of $X$. The probability density $\rho_{N}(y)$ of a gaussian element $Y$ is given by:

$$
\rho_{N}(y)=\frac{1}{\sigma \sqrt{2 \pi}} \exp \left[-\frac{1}{2}\left(\frac{y-m}{\sigma}\right)^{2}\right]
$$

To validate our model we compute the cumulative histogram of $X$. The match between empirical and theoret- 


\begin{tabular}{|c|c|c|c|c|}
\hline & lena & tree & baboon & water \\
\hline$\hat{\sigma}^{2}$ & 11.16 & 11.93 & 9.55 & 11.91 \\
\hline$\hat{m}$ & 0.08 & 0.11 & 0.05 & 0.18 \\
\hline
\end{tabular}

ESTIMATORS FOR DIFFERENT IMAGES.

\begin{tabular}{|c|c|c|c|c|}
\hline$P_{f a}$ & $10^{-4}$ & $10^{-5}$ & $10^{-6}$ & $10^{-7}$ \\
\hline$u$ & 3.71 & 4.26 & 4.75 & 5.19 \\
\hline$\eta\left(P_{f a}\right)$ & 12.85 & 14.75 & 16.45 & 17.97 \\
\hline \multicolumn{5}{|c}{ TABLE III }
\end{tabular}

$\eta\left(P_{f a}\right)$ FOR DIFFERENT FALSE RESPONSE PROBABILITIES.

ical probability densities is good (cf Fig.20). The law of the random variable $Y$ is consequently given by:

$$
P(X \leq x)=\Pi\left(\frac{y-m}{\sigma}\right)
$$

where

$$
\Pi(y)=\int_{-\infty}^{y} \rho_{N}(y)
$$

Using 100 different keys for each triangle of a given tes-

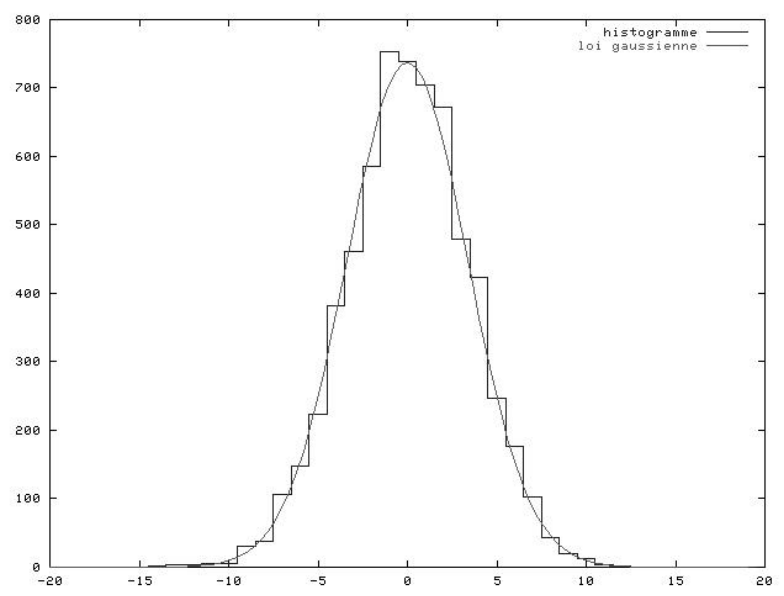

Fig. 20. Histogram of the value of correlation, in which the detection key is different from the insertion key. The results were obtained by using 100 keys per triangle. The continuous curve represents a gaussian probability density.

sellation, we have computed the estimate of the mean $\hat{m}$ and the variance $\hat{\sigma}^{2}$ (cf Table II).

$\eta\left(P_{f a}\right)$ is then computed from $\hat{\sigma}^{2}$ using the relation:

$$
\eta\left(P_{f a}\right)=u * \hat{\sigma}+\hat{m}
$$

The parameter $u$ corresponds to a normalized gaussian law. Table III represents different values $\eta\left(P_{f a}\right)$ depending on the false alarm probability.

7) A global decision is obtained using the global sum of $\operatorname{corr}\left(T_{w} ; \hat{T}_{w}\right)$.

The mean of the correlation calculated for each triangle of the tessellation allows also signature detection inside the image. In the case of a value greater than a threshold, the mark is detected.

We used the mean of the correlation to obtain a new statistic named $Z$ :

$$
Z=\frac{1}{N} \sum_{i=1, \ldots, N} \operatorname{corr}_{i}\left(T_{w} ; \hat{T}_{w}\right)
$$

It is a well-known result that $Z$ can be expressed by an empirical gaussian law of mean $\hat{m}_{Z}=\hat{m}$ and variance $\hat{\sigma}_{Z}^{2}=\hat{\sigma}^{2} / N$.

In addition, the mark can be also detected if:

$$
Z \geq \frac{\eta\left(P_{f a}\right)}{\sqrt{N}}
$$

8) The final decision is obtained from the different detection results.

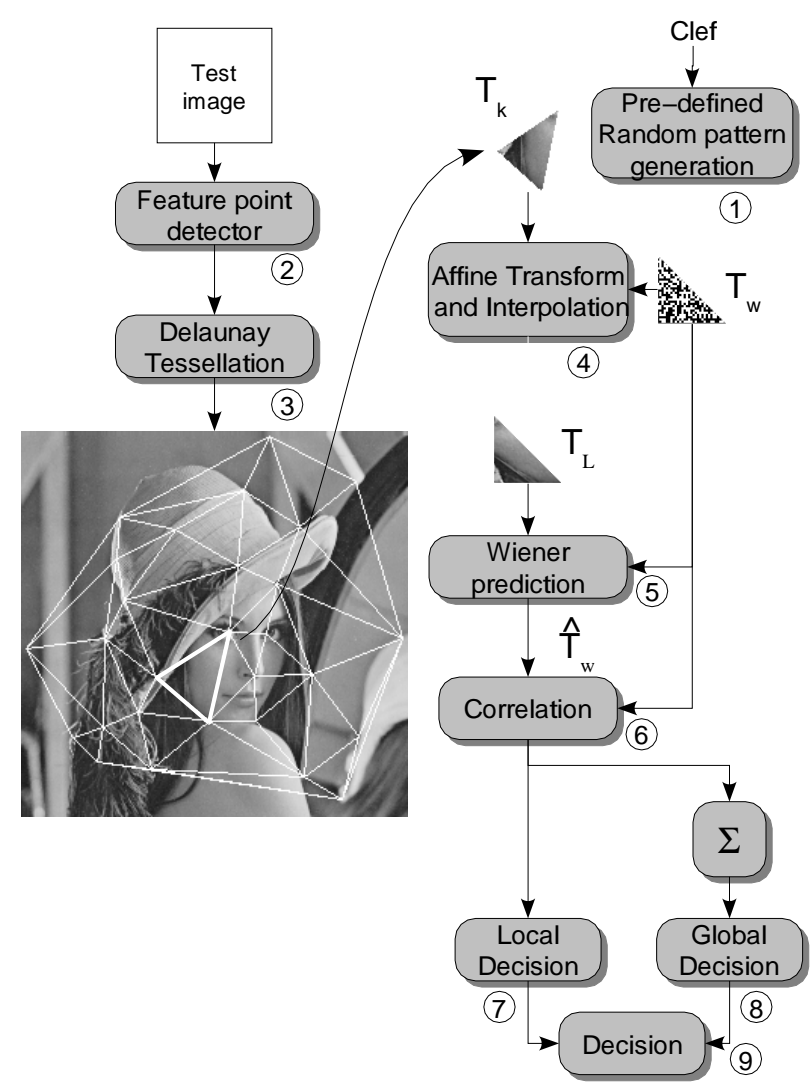

Fig. 21. Principle of the detection process

\section{Evaluation and Comparison}

The objective of this section is to show the capability of the algorithm to achieve good detection efficiency after geometrical transformations and classical process.

Ours tests were processed on 12 different images representing different classes of contents. The test database is depicted in Fig. 22.

Various categories of images have been chosen. Images baboon, lake, elaine, bridge include textured areas with high frequency components while the other images (plane, tank, 


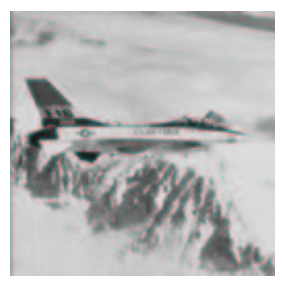

plane

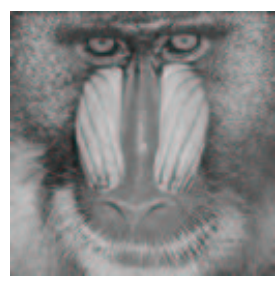

baboon

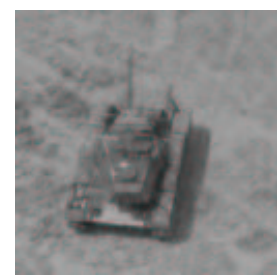

tank

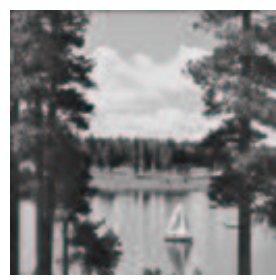

lake

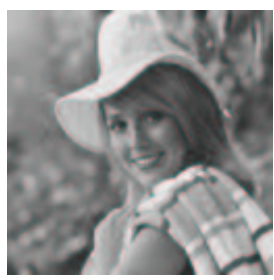

elaine

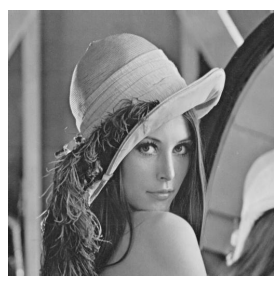

lena

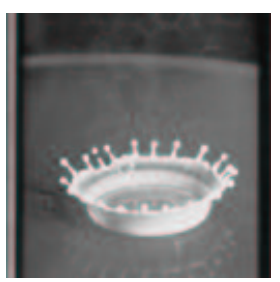

drop

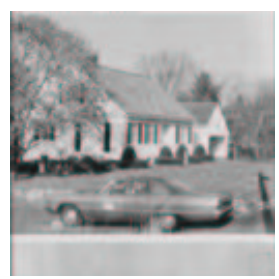

car

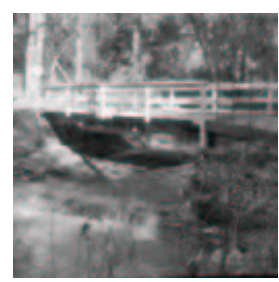

bridge

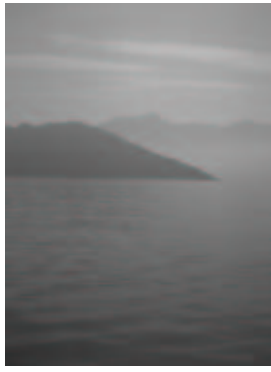

water

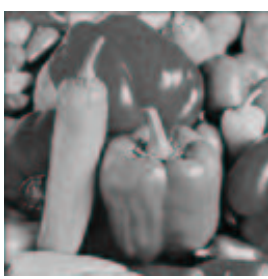

peppers

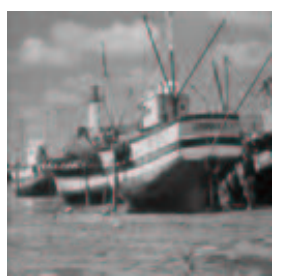

boat

Fig. 22. Images used in our tests.

\begin{tabular}{|c||c||c|c|c|c|c|c|c|c|c|c|c|c|}
\hline & & plane & baboon & tank & lake & elaine & lena & drop & car & bridge & water & peppers & boat \\
\hline \hline Stirmark & C.B.S. & OK & OK & OK & OK & OK & OK & OK & OK & OK & OK & OK & OK \\
\hline & Digimarc & Defeat & Defeat & Defeat & Defeat & Defeat & Defeat & Defeat & Defeat & Defeat & Defeat & Defeat & Defeat \\
\hline \hline Shearing & C.B.S. & OK & OK & OK & OK & OK & OK & OK & OK & OK & OK & OK & OK \\
\hline & Digimarc & Defeat & Defeat & Defeat & Defeat & Defeat & Defeat & Defeat & Defeat & Defeat & Defeat & Defeat & Defeat \\
\hline \hline Rot. 10 & C.B.S. & OK & OK & OK & OK & OK & OK & OK & OK & OK & OK & OK & OK \\
\hline & Digimarc & OK & OK & OK & OK & OK & OK & OK & OK & OK & OK & OK & OK \\
\hline \hline Scal. 80\% & C.B.S. & OK & Defeat & OK & OK & OK & OK & OK & OK & OK & OK & OK & OK \\
\hline & Digimarc & OK & OK & OK & OK & OK & OK & OK & OK & OK & OK & OK & OK \\
\hline \hline Scanning & C.B.S. & OK & Defeat & Defeat & OK & OK & OK & OK & OK & OK & Defeat & OK & OK \\
\hline & Digimarc & OK & OK & OK & OK & OK & OK & OK & OK & OK & OK & OK & OK \\
\hline \hline JPEG 50\% & C.B.S. & OK & OK & OK & OK & OK & OK & OK & OK & OK & OK & OK & OK \\
\hline & Digimarc & OK & OK & OK & OK & OK & OK & OK & OK & OK & OK & OK & OK \\
\hline
\end{tabular}

Benchmark RESUlts of THE CONTENT BASEd SCHEME (C.B.S.) AND COMPARison With DigimarC. "OK" MEANS THAT THE MARK HAS BEEN DETECTED (LOCAL AND/OR GLOBAL DETECTION)

lena, drop, car, bridge, water, peppers, cornouaille) include large homogeneous areas.

We tested the detector using a wide class of geometrical distortions and classical image processing operations (Jpeg compression and print and scan). Here is the list of the different process:

- the Stirmark distortion: this attack leads to a geometrical distortion that cannot be modelled by an affine transform on the whole image (cf section II-A). We have used the Stirmark 3.0 algorithm.

- Shearing transformation: we use the Gimp software to perform this transformation [10]. This filter can be considered as an affine transform.

- Scaling transform: we perform a scaling operation of $80 \%$.

- Rotation: we evaluated the robustness of the presented scheme for a rotation of $10^{\circ}$.

- Print and Scan: the print and scan test consists in printing the marked image on a classical laser printer and scanning it. In our test we used a $300 \times 300$ greyscale printer and a 300ppi scanner.

- JPEG compression: this compression scheme is one of the most used over the Internet. We have evaluated our scheme for a quality factor equal to $50 \%$. In this case, the compressed image has blocking artefacts.

Detection results are given on table IV. They indicate whether the global detection was successfully performed on the marked image or not. A false-alarm probability equal to $10^{-4}$ has been chosen. We also present the detection result obtained using the Digimarc watermarking tool available on Photoshop5.0. This commercial product is known for his robustness to a various category of filter. It uses template embedding to recover synchronisation after geometrical transformations[18].

1) StirMark: Our results illustrate the fact that the presented scheme is robust against the StirMark attack, the mark 


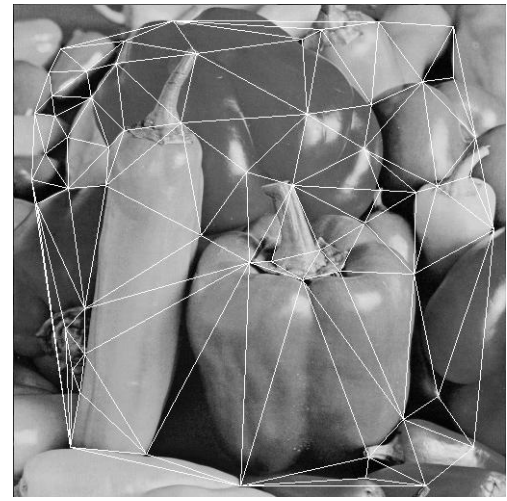

Original image and tessellation

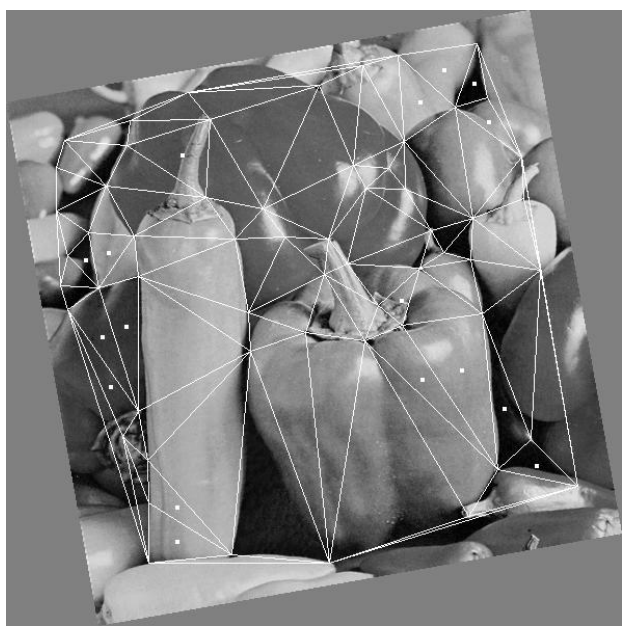

Marked image after rotation of $10^{\circ}$

Fig. 27. Effect of a $10^{\circ}$ on the marked peppers image. Global and Local detection have succedded.

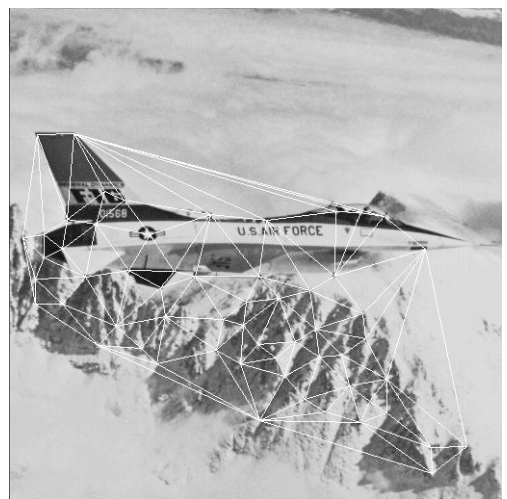

Original image and tessellation

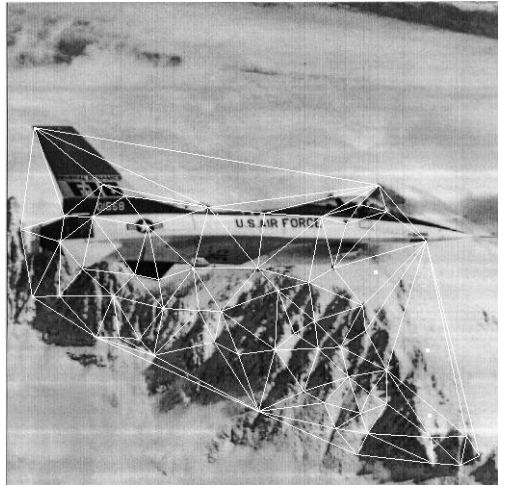

Marked image after print and scan

Fig. 28. Effect of the print and scan operation on the marked image plane. Global and Local detection have succeeded.

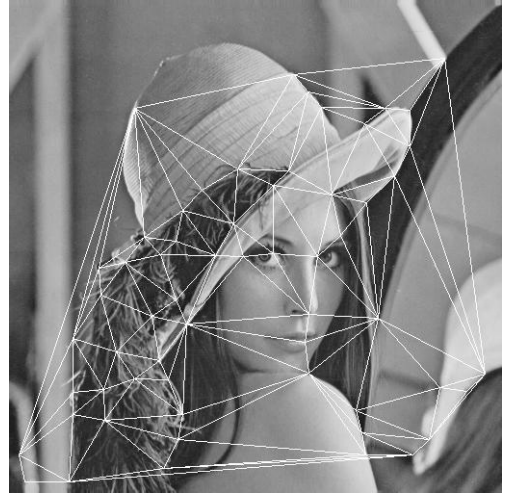

Original image and tessellation

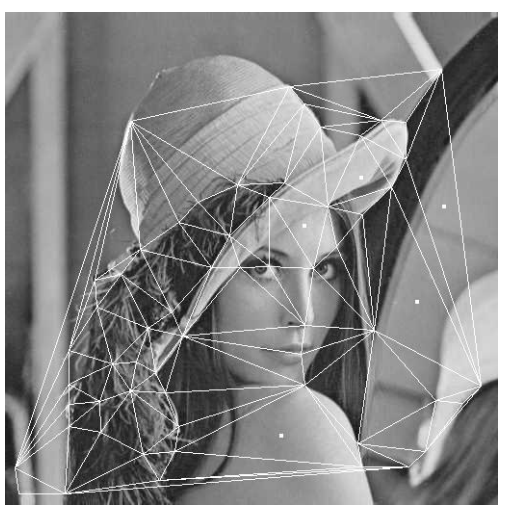

Marked image and Jpeg compression (50\%)

Fig. 29. Effect on Jpeg compression (50\%) on the marked lena image. Global and Local detection have succeeded.

cations. In AM'2001: Applications of Digital Image Processing, volume 4472 of SPIE Proceedings, San Diego, California USA, jul 2001.

[3] P. Bas, J-M. Chassery, and B. Macq. Robust watermarking based on the warping of pre-defined triangular patterns. In Proc. SPIE, pages 99-109, January 2000.

[4] B.Chen and G.W. Wornell. An information-theoretic approach to the design of robust digital watermarking systems. In Proceedings IEEE-
ICASSP'99, Phoenix (Arizona), March 1999.

[5] E. Bertin, S. Marchand-Maillet, and J-M. Chassery. Optimization in Voronoi Diagrams, pages 209-216. Kluwer Academic Publishers, 1994.

[6] B. Boufama, R. Mohr, and L. Morin. Using geometric properties for automatic object positioning. Image and Vision Computing, 16(1):27-33, 1998.

[7] J. Devars C. Achard-Rouquet, E. Bigorgne. Un detecteur de point carater- 
istiques sur des images multispectrales. extension vers un detecteur subpixellique. In GRETSI 99, pages 627-630, September 1999.

[8] J.-M. Chassery and A. Montanvert. Géométrie discrète en analyse d'images. Edition Hermès, 1991.

[9] I. Cox, J. Killian, T. Leighton, and T. Shamoon. Secure spread spectrum watermarking for multimedia. IEEE Transactions on Image Processing, 6(12):1673-1687, December 1997.

[10] M. Cutts. An introduction to the gimp. In ACM on Computer Graphics, http://www.acm.org/crossroads/xrds3-4/, July 1997.

[11] F. Davoine, P. Bas, P-A Hebert, and J-M Chassery. Watermarking et résistance aux déformations géométriques. In Coresa99, InstitutEurecom, Sophia Antipolis, France, June 1999.

[12] D. Delanay and B. Macq. Generalized 2-d cyclic patterns for secret watermark generation. In Proc. ICIP, volume 2, pages 77-80, Sept 2000.

[13] J. Dittmann, T. Fiebig, and R. Steinmetz. New approach for transformation-invariant image and video watermarking in the spatial domain: self-spanning patterns (ssp). In Ping Wah Wong and Edward J. Delp, editors, Electronic Imaging 2001: Security and Watermarking of Multimedia Content III, SPIE Proceedings, pages 176-186, San Jose, California USA, jan 2000.

[14] Y. Dufournaud, C. Schmid, and R. Horaud. Matching image with different resolutions. Computer Vision and Pattern Recognition, 1:612-618, 2000.

[15] J-L Dugelay and F.A.P. Petitcolas. Possible counter-attacks against geometric distortions. In Ping Wah Wong and Edward J. Delp, editors, EI'2000: Security and Watermarking of Multimedia Content II, volume 3971 of SPIE Proceedings, pages 358-370, San Jose, California USA, 23-28jan 2000.

[16] Z. Duric and N.F. Johnson. Recovering watermarks froms images. Technical report, Information and Software Engineering Tehcnical Report, San Diego, CA 92152-5000, April 1999.

[17] O. Faugeras, L. Quan, and P. Sturm. Self-calibration of a 1d projective camera and its application to the self-calibration of a $2 \mathrm{~d}$ projective camera. In Proceedings of the 5th European Conference on Computer Vision, Freiburg, Germany, pages 36-52, June 1998.

[18] B. Geoffrey. Photographic products and methods employing embedded information. Technical Report US patent 5,822,436, Digimarc Corporation, Lake Oswego, April 1996.

[19] C. Harris and M. Stephen. A combined corner and edge detector. In 4th Alvey Vision Conf, pages 147-151, 1988.

[20] F. Hartung, J.K. Su, and B. Girod. Spread spectrum watermarking: Malicious attacks and counter-attacks. In Proc. of SPIE: Security and Watermarking of Multimedia Contents, volume 3657, pages 147-158, San Jose CA, January 1999.

[21] J. R. Hernandez and F. Perez-Gonzalez. Statistical analysis of watermarking schemes for copyright protection of images. Proceedings of the IEEE, 87(7):1142-1143, july 1999.

[22] T. Kalker, G. Depovere, J. Haitsma, and M. Maes. A video watermarking system for broadcast monitoring. In Proc. SPIE, pages 103-112, January 1999.

[23] M. S. Kankanhalli, Rajmohan, and K.R. Ramakrishnan. Content-based watermarking of images. In Proc. of The 6th ACM International Multimedia Conference, pages 61-70, Bristol, UK, September 1998.

[24] R.G. Keys. Cubic convolution interpolation for digital image processing. In Proceedings of ICASSP, pages 1153-1160, December 1981.

[25] D. Kundur and D. Hatzinakos. Digital watermarking using multiresolution wavelet decomposition. In IEEE ICASSP'98, volume 5, pages 26592662, Seattle (USA), May 1998.

[26] M. Kutter. Watermarking resisting to translation, rotation and scaling. In Proc. of SPIE: Multimedia systems and applications, volume 3528, pages 423-431, Boston, November 1998.

[27] M. Kutter, S. K. Bhattacharjee, and T. Ebrahimi. Towards second generation watermarking schemes. In IEEE-ICIP'99, volume I, pages 320-323, Kobe (Japan), October 1999.

[28] C.-Y. Lin, J. A. Bloom, I. J. Cox, M. L. Miller, and Y.M. Lui. Rotation-, scale-, and translation-resilient public watermarking ofr images. In Proc. SPIE, pages 90-98, January 2000.

[29] E. Loupias, N. Sebe, S. Bres, and J.-M. Jolion. Wavelet-based salient points for image retrieval. In Proc. ICIP, to be published, Sept 2000.

[30] M.J.J. Maes and C.W.A. M. van Overveld. Digital watermarking by geometric warping. In IEEE-ICIP'98, volume II, pages 424-429, Chicago (IL, US), October 1998.

[31] D.P. Mitchel and A.N. Netravali. Reconstruction filters in computer graphics. Computer Graphics, 22(4):221-228, August 1988.

[32] H. Moravec. Obstacle avoidance and navigation in the real world by a seen robot rover. Technical Report CMU-RI-TR-3, Robotics Institute, Carnegie-Mellon University, September 1980.

[33] Joseph J. K. Ó Ruanaidh and Thierry Pun. Rotation, scale and translation signal spread spectrum digital image watermarking. Signa 66(3):303-317, May 1998.

[34] Shelby Pereira and Thierry Pun. Fast robust template matching for affine resistant image watermarking. In International Workshop on Information Hiding, volume LNCS 1768 of Lecture Notes in Computer Science, pages 200-210, Dresden, Germany, 29 September -1 October 1999. Springer Verlag.

[35] Fabien A.P. Petitcolas, Ross J. Anderson, and Markus G. Kuhn. Attacks on copyright marking systems. In David Aucsmith, editor, Information hiding: second international workshop, Lecture notes in computer science. Springer Verlag, Berlin, Germany, 1998.

[36] I. Pitas and T. Kaskalis. Applying signatures on digital images. In Workshop on Nonlinear Signal and Image Processing, pages 460-463, Neos Marmaras, June 1995. IEEE.

[37] F. Schmitt and H. Borouchaki. Algorithme rapide de maillage de delaunay dans Rd. In Springer, editor, Proc. des journées de géométrie algorithmique, pages 131-133, June 1990.

[38] S M Smith and J M Brady. Susan - a new approach to low level image processing. International Journal of Computer Vision, 23(1):45-78, may 1997.

[39] Q. Sun, J. Wu, and R. Deng. Recovering modified watermarked image with reference to originale image. In Proc. SPIE, pages 415-424, January 1999.

[40] A.Z. Tirkel, G. Rankin, R. Schyndel, and C.F. Osborne. Electronic water mark. In DICTA, pages 666-672, Austin (TX), Usa, December 1993.

[41] S. Voloshynovskiy, A. Herrigel, and Y. B. Rytsar. Watermark template attack. In Ping Wah Wong and Edward J. Delp, editors, EI'2001: Security and Watermarking of Multimedia Content III, SPIE Proceedings, San Jose, California USA, 22-25jan 2001.

[42] Koch E. Zhao J. Embedding robust labels into images for copyright protection. Technical report, Fraunhofer Institute for Computer Graphics, Darmstadt, Germany, 1994. 